\title{
Solving Two Dimensional Coupled Burger's Equations and Sine-Gordon Equation Using El-Zaki Transform-Variational Iteration Method
}

\author{
Marwa H. Al-Tai ${ }^{1,2, *}$ and Ali Al-Fayadh ${ }^{1}$ \\ ${ }^{1}$ Department of Mathematics and Computer Applications, College of Science, Al-Nahrain University, Baghdad, Iraq \\ ${ }^{2}$ Iraqi Ministry of Education, Second General Directorate of Education Al-Karkh in Baghdad, Iraq
}

\begin{tabular}{ll}
\hline \multicolumn{1}{c}{ Article's Information } & \multicolumn{1}{c}{ Abstract } \\
\hline Received: & In this paper, the combined form of the Elzaki transform and variation \\
$23-04-2021$ & iteration method is implemented efficiently in finding the analytical and \\
Accepted: & numerical solutions of the two-dimensional nonlinear coupled Burger's partial \\
$29-05-2021$ & differential equations and sine-Gordon partial differential equation. The \\
Published: & obtained solutions were compared to the exact solutions and other existing \\
$27-06-2021$ & methods. Illustrative examples show the efficiency and the power of the used
\end{tabular}

DOI: 10.22401/ANJS.24.2.07

*Corresponding author: Marwahazim8585@gmail.com

\section{Introduction}

Differential equations (DEs) play a major role in describing a wide range of natural phenomena undergoing change. They relate an unknown function to its derivatives. These functions usually represent physical quantities whereas the derivatives represent their rates of change. Partial Differential Equations (PDEs) as well as Ordinary Differential Equations (ODEs) usually arise in many diverse applications, such as fluid flow, mechanical systems, physics, and relativity [1]. There are a variety of numerical and analytical techniques for solving the PDEs.

The analytical solution for a given PDE is always preferable due to the limitation of the numerical solutions that cannot tell us much about the qualitative behavior of systems. However in many instances, systems described by PDEs are often so complex or so large where a purely analytical solution to the equations is not tractable, or many of the modeled problems have resulted in nonlinear PDEs in which the exact solutions are difficult to obtain by using analytical methods. In this case, the numerical methods are needed to be developed for solving DEs despite these methods have some errors.

Many numerical methods were developed over a period of time like the finite difference method [2], then the finite element method [3], after that finite volume method [4], more than that boundary element method [5], and mesh free method [6], finally the spectral method [7], in order to find more accurate approximation solutions to these nonlinear equations. On the other hand, studying the Localization in a small part of space, which is often described by nonlinear equations, plays a major role in various fields like plasma physics, hydrodynamic, nonlinear optic, etc. The exact solutions of these non-linear equations are very important. Many researchers had paid great interest to study solutions of non-linear equations by using several methods, like Darboux transformation [8], Backland transformation [9], [10], the inverse scattering method [11], the tanh method [12], the sine-cosine method [13], and Hirota's bilinear method [14].

Burger's equation is a fundamental PDE in fluid mechanics [15] and in the Burger's model of turbulence [16]. This equation often occurs in various areas of applied mathematics, like the modeling of dynamics, the acoustic waves, and the heat conduction. It is solved analytically for arbitrary initial conditions [17].

The coupled Burger's equations are of rich dynamics. These equations are derived by Esipov [18]. The following system is known as the $(1+1)$-coupled Burger's system:

$$
\begin{aligned}
& u_{t}-u_{x x}-2 u u_{x}+(u v)_{x}=0 \\
& v_{t}-v_{x x}-2 v v_{x}+(u v)_{x}=0
\end{aligned}
$$

This system may be considered as a normal model of sedimentation and evolution of scaled volumes concentrations of two kinds of particles in fluid suspensions and colloids under the effect of gravity. Many authors have proposed various numerical methods to solve the above system. Kaya [19] used Adomian decomposition 


\section{Al-Nahrain Journal of Science}

ANJS, Vol.24 (2), June, 2021, pp. 41-47

method (ADM). In [20], the variational iteration method (VIM) was successfully used for solving and analyzing this system. In [21], a modified extended hyperbolic tangent-function method was implemented, while numerical solutions to this system of equations have been tried to getting by many authors. In [22], the differential transform method was employed to get the numerical solution of this system. The above one-dimensional system of Burger's equations was solved by collocation method based on the cubic B-spline [23]. Srivastava et al. [24] have applied an implicit logarithmic finite difference method for numerical solution of the above onedimensional coupled non-linear Burger's equation. In [25], the Variational Iteration Transform Method (VITM) was employed to determine the solution of the $(1+1)$-coupled Burger's equations.

On the other hand, the following two-dimensional system of nonlinear PDEs is known as the twodimensional Burger's equation:

$$
\begin{aligned}
& u_{t}+u u_{\mathrm{\gamma}}+v u_{y}=\frac{1}{R e}\left(u_{\mathrm{X}}+u_{y y}\right) \\
& v_{t}+u v_{\mathrm{X}}+v v_{y}=\frac{1}{R e}\left(v_{\mathrm{X}}+v_{y y}\right)
\end{aligned}
$$

where $(\mathrm{x}, y) \in M, t>0$ and subject to the initial condition

$$
\boldsymbol{u}(\mathrm{x}, \boldsymbol{y}, \mathbf{0})=\boldsymbol{g}(\mathrm{x}, \boldsymbol{y}),(\mathrm{x}, \boldsymbol{y}) \in \boldsymbol{M}
$$$$
\boldsymbol{v}(\mathrm{x}, \boldsymbol{y}, \mathbf{0})=\boldsymbol{h}(\mathrm{x}, \boldsymbol{y}),(\mathrm{x}, \mathrm{y}) \in \boldsymbol{M}
$$

where:

$$
\boldsymbol{M}=\{(\mathrm{x}, \boldsymbol{y}): \boldsymbol{a} \leq \boldsymbol{x} \leq \boldsymbol{b}, \boldsymbol{a} \leq \boldsymbol{y} \leq \boldsymbol{b}\}
$$

$u(\mathrm{x}, y, t)$ and $v(\mathrm{x}, y, t)$ are the velocity components to be determined, and $\mathrm{g}, h$ are known functions, and $R e$ is the Reynolds number.

Many authors have been proposed various numerical methods to solve the system of two-dimensional coupled Burger's equation. In 1978, Jain and Holla [26] proposed two algorithms based on cubic spline function technique to solve system of the coupled Burger's equations, while Arminjon P. and Beauchamp C. [27] had developed a finite-element method using rectangular for solving the same system. Due to the unavailability of the exact solutions of this system, the precision of these methods was assessed indirectly via grid refinement. The twodimensional Hopf-Cole transformation had been applied by Fletcher [28] to overcome this problem. This transformation generates the exact solutions of some specified twodimensional Burger's equation, for that the accuracy of these numerical methods may be tested. In [29], Bahadir A.R. utilized central finite-difference method in the spatial dimension and backward Euler scheme in the temporal dimension to solve the system of two- dimensional Burger's equation. In 2008, A.H. Khater et al. solved the system of two-dimensional Burger's equations using the Chebyshev spectral collocation methods [30]. Multiple kink solutions had been used by Wazwaz to solve and analyzed this system [31]. In [32], the variational iteration method was applied to solve the system of twodimensional Burger's equation. In 2010, Wenyuan Liao solved the system of two-dimensional Burger's equation using the fourth order finite difference method [33]. In
2011, the local discontinuous Galerkin the finite element method based on Hopf-Cole transformations were used for finding numerical solutions of the system of twodimensional Burger's equation [34]. In 2011, lattice Boltzmann method had been used by Fang Liu and Weiping Shi for solving the system of two-dimensional Burger's equation [35], while in [36], the homotopy perturbation method based on the Pade approximation was proposed to solve this system. In 2012, Hossein Aminikhah had introduced a new hybrid of the Laplace transform method and the new homotopy perturbation method (LTNHPM) for solving the system of twodimensional Burger's equation [37]. Mohammad Vaghefi et al. in 2014 used finite difference method to solve this system of equations [38]. In [39], the author proposed the Laplace Decomposition Method (LDM) to solve the twodimensional non-linear Burger's DEs.

In addition, one of the important non-linear partial differential equations that arise in different fields of sciences is the sine-Gordon equation. It is a non-linear hyperbolic partial differential equation, its applications appear in differential geometry, and it has many applications in propagation of fluxons in Josephson junctions [40], solid state physics, nonlinear optics, stability of fluid motions, dislocations in crystals stability of fluid motions, the motion of a rigid pendulum attached to a stretched wire, dislocations in crystals [41], and there other applications in scientific field. For this important application there are great attempts to getting different solutions and physical phenomena related to this equation [42], such as the modified decomposition method [43] [44], tanh method [45], modified homotopy perturbation method [46], homotopy-perturbation method [47], the variational iteration method [48], the combined Laplace transform-Variation iteration method [ 49 ], modified variation iteration method [50], modified decomposition Method [51] and homotopy analysis method [52].

The variational iteration method (VIM) was first proposed by He [53], [54], [55], [56] and was successfully applied for solving ODEs, such as autonomous ODE in [57], nonlinear oscillations [58] and other fields. The VIM has been used to solve linear or non-linear ODEs and PDEs without need small parameter or linearization and by few iterations lead to high accurate solutions.

In this paper, we extend the variational iteration transformed method (VITM) to combine the well-known El-Zaki transform and the VIM which is denoted by (VITM) for solving the system of two-dimensional coupled Burger's equation and sine-Gordon equation. The numerical results obtained by (VITM) of the test problems are compared to the exact solution and the solutions by some of those already existing methods in the literature. The obtained numerical solutions by the proposed (VITM) are compatible with the exact solutions. 


\section{Al-Nahrain Journal of Science}

ANJS, Vol.24 (2), June, 2021, pp. 41-47

2. General Mechanism of El-Zaki Transform and Variational Iteration Method

El-Zaki transform was defined by El-Zaki in [59], [60] as a new integral transform called El-Zaki transform defined for functions of exponential order, is proclaimed. We consider functions in the set $\mathrm{A}$ defined by:

$$
\begin{gathered}
A=\left\{f(t)\left|\exists M, B_{1}, B_{2}>0\right| f(t)<M e^{\frac{|t|}{k_{j}}}, \text { if } t \in\right. \\
\left.(-1)^{j} X[0, \infty)\right\}
\end{gathered}
$$

For a given function in the set $\mathrm{A}$, the constant $\mathrm{M}$ must be finite number, $B_{1}, B_{2}$ may be finite or infinite.

The El-Zaki transform denoted by the operator (E) defined by the integral equations:

$$
\begin{aligned}
E[f(t)] & =w^{2} \int_{0}^{\infty} f(w t) e^{-t} d t \\
& =T(w), w \in\left(B_{1}, B_{2}\right)
\end{aligned}
$$

ELzaki transform was shown to have united preserving properties, and hence may be used to solve problems without resorting to the frequency domain. As will be seen below, this is one of the advantages of this new transform, particularly in applications to problems with physical dimensions. In fact El-Zaki transform which is itself linear preserves linearity [61].

As we solving PDEs then we need to differentiation formula of El-Zaki transform:

$$
\left.\begin{array}{c}
E\left[f^{\prime}(t)\right]=\frac{T(w)}{w}-w f(0) \\
E\left[f^{\prime \prime}(t)\right]=\frac{T(w)}{w^{2}}-f(0)-w f^{\prime}(0) \\
\vdots \\
E\left[f^{n}(t)\right]=\frac{T(w)}{w^{n}}-\sum_{k=0}^{n-1} w^{2-n+k} f^{(k)}(0)
\end{array}\right\}
$$

Now, after we propose El-Zaki transform moving to VIM, the following nonlinear partial differential equation will be used to demonstrate the basic concept of the VIM:

$$
\boldsymbol{L u}+\boldsymbol{N u}=\boldsymbol{f}(\boldsymbol{x})
$$

where $L$ is a linear operator, $N$ is a nonlinear operator and $f$ is an inhomogeneous term, which is an analytical function. According to VIM, we can construct a correction functional for eq. above as follows:

$$
\begin{gathered}
u_{n+1}(x)=u_{n}(x)+\int_{0}^{x} \lambda(s)\left(L u_{n}(s)+N \widetilde{u}_{n}(s)-\right. \\
f(s)) d s
\end{gathered}
$$

Where $\lambda$ is a Lagrange multiplier, and the solution is given by $u=\lim _{n \rightarrow \infty} u_{n}$.

\section{Illustrative Examples}

In this section we take three examples, Example 1 represent system of two-dimensional couple Burger's equation, while Examples 2 and 3 represent the sineGordon equation.

Example 1. Consider the system of the two-dimensional Couple Burger's Equation

$$
\begin{aligned}
& u_{t}+u u_{x}+v u_{y}=\frac{1}{R e}\left(u_{x x}+u_{y y}\right) \\
& v_{t}+u v_{x}+v v_{y}=\frac{1}{R e}\left(v_{x x}+v_{y y}\right)
\end{aligned}
$$

$$
\begin{aligned}
& u(x, y, 0)=x+y \\
& v(x, y, 0)=x-y
\end{aligned}
$$

First take El-Zaki transform to equations (10) and (11) both sides:

$$
\begin{gathered}
E\left[u_{t}\right]+E\left[u u_{x}+v u_{y}\right]=E\left[\frac{1}{R e}\left(u_{x x}+u_{y y}\right)\right] \\
E\left[v_{t}\right]+E\left[u v_{x}+v v_{y}\right]=E\left[\frac{1}{R e}\left(v_{x x}+v_{y y}\right)\right] \\
\frac{U(x, y, w)}{w}-w u(x, y, 0)+E\left[u u_{x}+v u_{y}\right]= \\
E\left[\frac{1}{R e}\left(u_{x x}+u_{y y}\right)\right] \\
\frac{v(x, y, w)}{w}-w v(x, y, 0)+E\left[u v_{x}+v v_{y}\right]= \\
E\left[\frac{1}{R e}\left(v_{x x}+v_{y y}\right)\right]
\end{gathered}
$$

By multiplying equations (16) and (17) by $w$ and substitute the initial condition in (12) and (13)

$$
\begin{gathered}
U(x, y, s)-w^{2}[x+y]+w L\left[u u_{x}+v u_{y}\right]= \\
w L\left[\frac{1}{R e}\left(u_{x x}+u_{y y}\right)\right] \\
V(x, y, s)-w^{2}[x-y]+w L\left[u v_{x}+v v_{y}\right]= \\
w L\left[\frac{1}{R e}\left(v_{x x}+v_{y y}\right)\right]
\end{gathered}
$$

Now taking El-Zaki inverse to equations (18) and (19):

$$
\begin{gathered}
u(x, y, t)-[x+y]+E^{-1}\left[w E\left[u u_{x}+v u_{y}\right]\right]= \\
E^{-1}\left[w E\left[\frac{1}{R e}\left(u_{x x}+u_{y y}\right)\right]\right] \\
v(x, y, t)-[x-y]+E^{-1}\left[w E\left[u v_{x}+v v_{y}\right]\right]= \\
E^{-1}\left[w E\left[\frac{1}{R e}\left(v_{x x}+v_{y y}\right)\right]\right]
\end{gathered}
$$

Derivative eq. (20 and (21) by $\frac{\partial}{\partial t}$

$$
\begin{gathered}
u_{t}(x, y, t)+\frac{\partial}{\partial t}\left\{E^{-1}\left[w E\left[u u_{x}+v u_{y}\right]\right]\right\}= \\
\frac{\partial}{\partial t}\left\{E^{-1}\left[w E\left[\frac{1}{R e}\left(u_{x x}+u_{y y}\right)\right]\right]\right\} \\
v_{t}(x, y, t)+\frac{\partial}{\partial t}\left\{E^{-1}\left[w E\left[u v_{x}+v v_{y}\right]\right]\right\}= \\
\frac{\partial}{\partial t}\left\{E^{-1}\left[w E\left[\frac{1}{R e}\left(v_{x x}+v_{y y}\right)\right]\right]\right\} \\
u_{t}(x, y, t)+\frac{\partial}{\partial t}\left\{E^{-1}\left[w E\left[u u_{x}+v u_{y}\right]\right]\right\}- \\
\frac{\partial}{\partial t}\left\{E^{-1}\left[w E\left[\frac{1}{R e}\left(u_{x x}+u_{y y}\right)\right]\right]\right\}=0 \\
v_{t}(x, y, t)+\frac{\partial}{\partial t}\left\{E^{-1}\left[w E\left[u v_{x}+v v_{y}\right]\right]\right\}- \\
\frac{\partial}{\partial t}\left\{E^{-1}\left[w E\left[\frac{1}{R e}\left(v_{x x}+v_{y y}\right)\right]\right]\right\}=0
\end{gathered}
$$

By the correction function of the irrational method:

$$
\begin{gathered}
u_{n+1}(x, y, t)=u_{n}(x, y, t)+\int_{0}^{t} \lambda_{1}\left\{\left(u_{n}\right)_{\xi}(x, y, \xi)+\right. \\
\frac{\partial}{\partial t}\left\{E^{-1}\left[w E\left[u_{n}\left(u_{n}\right)_{x}+v_{n}\left(u_{n}\right)_{y}(x, y, \xi)\right]\right]\right\}+ \\
\left.\frac{\partial}{\partial t}\left\{E^{-1}\left[w E\left[\frac{1}{R e}\left(\left(u_{n}\right)_{x x}+\left(u_{n}\right)_{y y}\right)\right]\right]\right\}\right\} d \xi \ldots \\
v_{n+1}(x, y, t)=v_{n}(x, y, t)+\int_{0}^{t} \lambda_{2}\left\{\left(v_{n}\right)_{\xi}(x, y, \xi)+\right. \\
\frac{\partial}{\partial t}\left\{E^{-1}\left[w E\left[u_{n}\left(v_{n}\right)_{x}+v_{n}\left(v_{n}\right)_{y}(x, y, \xi)\right]\right]\right\}+ \\
\left.\frac{\partial}{\partial t}\left\{E^{-1}\left[w E\left[\frac{1}{R e}\left(\left(v_{n}\right)_{x x}+\left(v_{n}\right)_{y y}\right)\right]\right]\right\}\right\} d \xi \ldots(27)
\end{gathered}
$$

Then take $n=0$ to find $u_{1}$ and $\lambda_{1}=\lambda_{2}=-1$ 


\section{Al-Nahrain Journal of Science}

ANJS, Vol.24 (2), June, 2021, pp. 41-47

$$
\begin{aligned}
& u_{1}(x, y, t)=u_{0}(x, y, t)-\int_{0}^{t}\left\{\left(u_{0}\right)_{\xi}(x, y, \xi)+\right. \\
& \frac{\partial}{\partial t}\left\{E^{-1}\left[w E\left[u_{0}\left(u_{0}\right)_{x}+v_{0}\left(u_{0}\right)_{y}(x, y, \xi)\right]\right]\right\}+ \\
& \left.\frac{\partial}{\partial t}\left\{E^{-1}\left[w E\left[\frac{1}{R e}\left(\left(u_{0}\right)_{x x}+\left(u_{0}\right)_{y y}\right)\right]\right]\right\}\right\} d \xi \ldots \\
& v_{1}(x, y, t)=v_{0}(x, y, t)-\int_{0}^{t}\left\{\left(v_{0}\right)_{\xi}(x, y, \xi)+\right. \\
& \frac{\partial}{\partial t}\left\{E^{-1}\left[w E\left[u_{0}\left(v_{0}\right)_{x}+v_{0}\left(v_{0}\right)_{y}(x, y, \xi)\right]\right]\right\}+ \\
& \left.\frac{\partial}{\partial t}\left\{E^{-1}\left[w E\left[\frac{1}{R e}\left(\left(v_{0}\right)_{x x}+\left(v_{0}\right)_{y y}\right)\right]\right]\right\}\right\} d \xi \ldots \\
& u_{1}(x, y, t)=(x+y)-(2 x t) \\
& v_{1}(x, y, t)=(x-y)-(2 y t)
\end{aligned}
$$

The values of the component $u_{1}$ obtained by the used VITM and comparing with the exact solution, the VIM, LDM, and EHPM respectively; are showed in Tables 1 and (2), while Figures 1 and 2 depicted the results of Tables 1 and 2 , respectively.

Table 1. The results of $u_{1}$ compared to the exact solution and the VITM and LDM, where $R e=1, t=0.1, y=0.1$.

\begin{tabular}{ccccc}
\hline $\boldsymbol{x}$ & Exact & VITM & Error & LDM \\
\hline 0.1 & 0.184 & 0.18 & 0.004 & 0.004 \\
0.2 & 0.265 & 0.26 & 0.005 & 0.006 \\
0.3 & 0.347 & 0.34 & 0.007 & 0.008 \\
0.4 & 0.429 & 0.42 & 0.009 & 0.01 \\
0.5 & 0.51 & 0.5 & 0.01 & 0.012 \\
\hline
\end{tabular}

Table 2. The results of $u_{1}$ compared to the exact solution and the VITM and EHPT, where $R e=1, x=0.2, y=0.2$.

\begin{tabular}{ccccc}
\hline $\boldsymbol{t}$ & Exact & VITM & Error & EHPM \\
\hline 0.05 & 0.382 & 0.38 & 0.002 & 0.19055 \\
0.1 & 0.367 & 0.36 & 0.007 & 0.1833 \\
0.15 & 0.356 & 0.34 & 0.006 & 0.178 \\
0.2 & 0.348 & 0.32 & 0.008 & 0.171 \\
0.25 & 0.343 & 0.3 & 0.043 & 0.172 \\
\hline
\end{tabular}

The values of the component $v_{1}$ obtained by the used VITM and comparing with the exact solution, the VIM, LDM, and EHPM respectively; are showed in Tables 3 and 4, while Figures 3 and 4 depicted the results of Table 3 and Table 4 respectively.

Table 3. The results of $v_{1}$ compared to the exact solution and the VITM and LDM, where $R e=1, t=0.1, y=0.1$.

\begin{tabular}{ccccc}
\hline $\mathrm{X}$ & Exact & VITM & Error & LDM \\
\hline 0.1 & -0.02 & -0.02 & 0 & 0 \\
0.2 & 0.082 & 0.08 & 0.002 & 0.002 \\
0.3 & 0.184 & 0.18 & 0.004 & 0.004 \\
0.4 & 0.286 & 0.28 & 0.006 & 0.006 \\
0.5 & 0.388 & 0.38 & 0.008 & 0.008 \\
\hline
\end{tabular}

Table 4. The results of $v_{1}$ compared to the exact solution and the VITM and EHPT, where $R e=1, x=0.2, y=0.2$.

\begin{tabular}{ccccc}
\hline $\boldsymbol{t}$ & Exact & VITM & Error & EHPM \\
\hline 0.05 & -0.02 & -0.02 & 0 & -0.01 \\
0.1 & -0.041 & -0.04 & 0.001 & -0.02 \\
0.15 & -0.063 & -0.06 & 0.003 & -0.04 \\
0.2 & -0.087 & -0.08 & 0.007 & -0.043 \\
0.25 & -0.114 & -0.1 & 0.014 & -0.067 \\
\hline
\end{tabular}

Example 2. We consider the following sine-Gordon equation:

$$
u_{t t}-u_{x x}+\sin u=0
$$

Subject to the initial conditions:

$$
u(x, 0)=0, u_{t}(x, 0)=4 \operatorname{sech}(x)
$$

The exact solution is:

$$
u(x, t)=4 \tan ^{-1}(t \operatorname{sech}(x))
$$

Taking El-Zaki transform to equation (10) both sides:

$$
E\left[u_{t t}\right]-E\left[u_{x x}\right]+E[\sin u]=0
$$

$$
\frac{T(w)}{w^{2}}-f(x, 0)-w f^{\prime}(x, 0)-E\left[u_{x x}\right]+E[\sin u]=0
$$

By multiply equation (14) by $\mathrm{w}^{2}$ we get:

$$
\begin{aligned}
& T(w)-w^{2} f(x, 0)-w^{3} f^{\prime}(x, 0)-w^{2} E\left[u_{x x}\right]+ \\
& w^{2} E[\sin u]=0
\end{aligned}
$$

Now, using initial condition:

$$
\begin{aligned}
& T(w)-0-w^{3}(4 \operatorname{sech}(x))-w^{2} E\left[u_{x x}\right]+ \\
& w^{2} E[\sin u]=0 \\
& T(w)=w^{3}(4 \operatorname{sech}(x))+w^{2} E\left[u_{x x}\right]-w^{2} E[\sin u]
\end{aligned}
$$

Taking inverse of El-Zaki transform to equation (17)

$$
\boldsymbol{u}(\boldsymbol{x}, \boldsymbol{t})=\boldsymbol{t}(4 \operatorname{sech}(x))+E^{-1}\left\{w^{2} E\left[u_{x x}\right]\right\}-
$$

$$
E^{-1}\left\{w^{2} E[\sin u]\right\}
$$

Now, differentiating equation (18) with respect to $t$

$$
\begin{aligned}
u_{t}(x, t)= & (4 \operatorname{sech}(x))+\frac{\partial}{\partial t} E^{-1}\left\{w^{2} E\left[u_{x x}\right]\right\}- \\
& \frac{\partial}{\partial t} E^{-1}\left\{w^{2} E[\sin u]\right\}
\end{aligned}
$$

Using the VIM

$$
\begin{aligned}
u_{n+1}(x, t) & =u_{n}(x, t)+\int_{0}^{t}(s-t)\left(\left(u_{n}\right)_{\xi}(x, \xi)-\right. \\
& 4 \operatorname{sech}(x)-\frac{\partial}{\partial t} E^{-1}\left\{w^{2} E\left[\left(u_{n}\right)_{x x}(x, \xi)\right]\right\}+ \\
& \left.\frac{\partial}{\partial t} E^{-1}\left\{w^{2} E\left[\sin u_{n}(x, \xi)\right]\right\}\right) d \xi
\end{aligned}
$$

To find $u_{1}$, take $n=0$

$$
\begin{aligned}
u_{1}(x, t)= & u_{0}(x, t)+\int_{0}^{t}(s-t)\left(\left(u_{0}\right)_{\xi}(x, \xi)-\right. \\
& 4 \operatorname{sech}(x)-\frac{\partial}{\partial t} E^{-1}\left\{w^{2} E\left[\left(u_{0}\right)_{x x}(x, \xi)\right]\right\}+ \\
& \left.\frac{\partial}{\partial t} E^{-1}\left\{w^{2} E\left[\sin u_{0}(x, \xi)\right]\right\}\right) d \xi \\
= & 4 t \operatorname{sech}(x)+\int_{0}^{t}(s-t)(4 \operatorname{sech}(x)- \\
& 4 \operatorname{sech}(x)-\frac{\partial}{\partial \xi} E^{-1}\left(w ^ { 2 } 4 \xi \left(-\operatorname{sech}^{3}(x)+\right.\right. \\
& \left.\left.\tanh ^{2}(x) \operatorname{sech}(x)\right)\right)+ \\
& \frac{\partial}{\partial \xi} E^{-1}\left(w^{2} E\{\sin (4 \xi \operatorname{sech}(x))\}\right) d \xi \ldots(44)
\end{aligned}
$$




\section{Al-Nahrain Journal of Science}

ANJS, Vol.24 (2), June, 2021, pp. 41-47

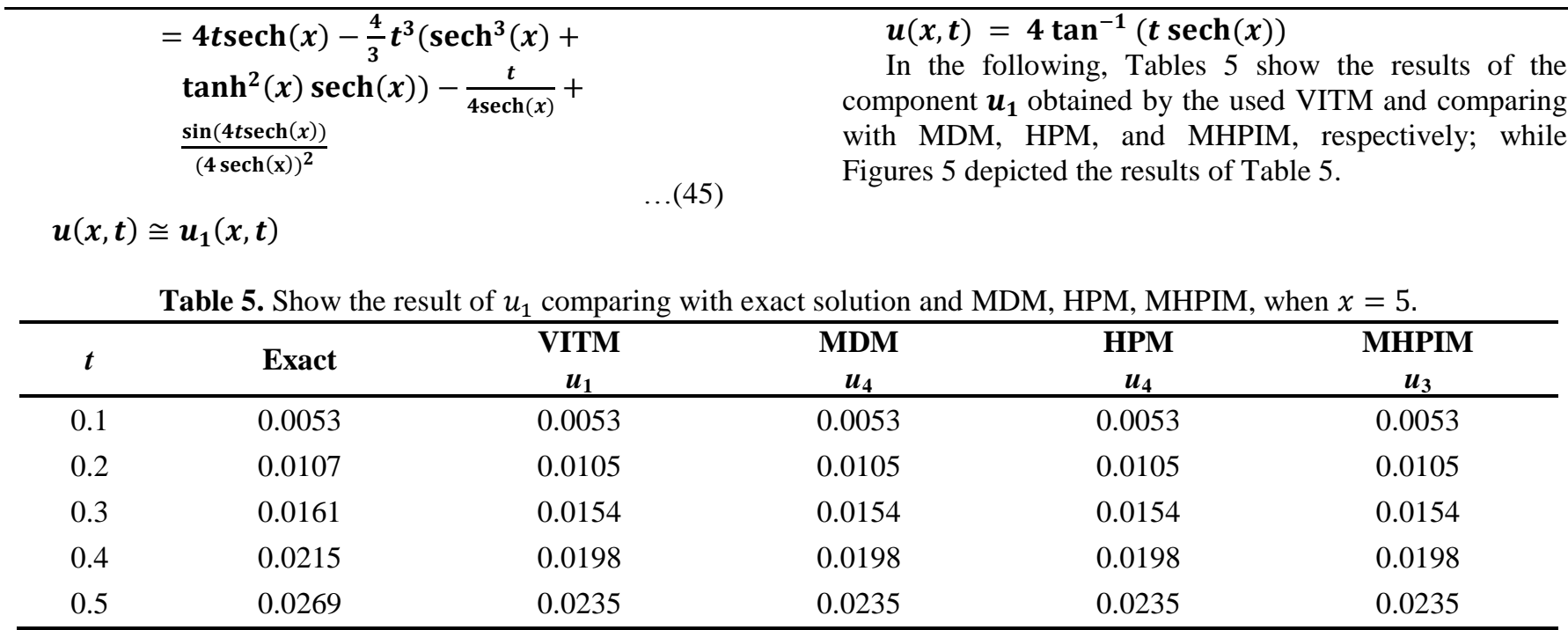

It is noted that the exact solution can be obtained for any $x$ and $t$ values from the first iteration by using the VITM method, while the modified decomposition method and the homotopy perturbation method have obtained the exact solutions from the 4-order approximate solution. In addition, the modified homotopy perturbation method has obtained the exact solutions from the 3 -order approximate solution.

Example 3. Consider the following sine-Gordon equation:

$$
u_{t t}-u_{x x}=\sin u
$$

Subject to the initial conditions:

$$
\boldsymbol{u}(\boldsymbol{x}, \mathbf{0})=\pi+\eta \cos \beta x, u_{t}(x, 0)=0
$$

By applied equation (19), we get:

$$
\boldsymbol{u}_{t}(\boldsymbol{x}, \boldsymbol{t})-\frac{\partial}{\partial t}\left\{E^{-1} \boldsymbol{w}^{2} E\left[\boldsymbol{u}_{x x}\right]\right\}+\frac{\partial}{\partial t}\left\{E^{-1} \boldsymbol{w}^{2} E[\sin u]\right\}=0
$$

By the correction function:

$$
\begin{aligned}
\boldsymbol{u}_{\mathbf{1}}= & \boldsymbol{u}_{\mathbf{0}}+\int_{\mathbf{0}}^{\boldsymbol{t}}(\boldsymbol{s}-\boldsymbol{t})\left(\left(\boldsymbol{u}_{\mathbf{0}}\right)_{\xi}(\boldsymbol{x}, \xi)-\frac{\boldsymbol{\partial}}{\boldsymbol{\partial} \boldsymbol{t}}\left\{\boldsymbol{E}^{-\mathbf{1}} \boldsymbol{w}^{\mathbf{2}}\right.\right. \\
& \left.\boldsymbol{E}\left[\left(\boldsymbol{u}_{\mathbf{0}}\right)_{x \boldsymbol{x}}\right]+\frac{\boldsymbol{\partial}}{\boldsymbol{\partial} \boldsymbol{t}}\left\{\boldsymbol{E}^{-\mathbf{1}} \boldsymbol{w}^{\mathbf{2}} \boldsymbol{E}\left[\sin \boldsymbol{u}_{\mathbf{0}}\right]\right\}\right) \boldsymbol{d} \xi \\
= & \pi+\eta \cos (\beta x)-\frac{t^{3}}{3}\left[[\eta \cos (\beta x)] \cdot \beta^{2}-\right. \\
& \sin (\eta \cos (\beta x))]
\end{aligned}
$$

Hence $\boldsymbol{u}(\boldsymbol{x}, \boldsymbol{t})=\lim _{n \rightarrow \infty} \widetilde{\boldsymbol{u}}_{n}$ and therefore $\boldsymbol{u}(\boldsymbol{x}, \boldsymbol{t}) \cong$ $u_{1}(x, t)$.

To explain result of this example where $\beta=\frac{\sqrt{2}}{2}$ and $\eta=$ constant. The VITM gives the same approximation solution of sine-Gordon equation from the first iteration, while the Modified Decomposition Method gives the same approximation solution from the three-order [1], and the Reduced Differential Transform Method gives the approximation solution from the $5^{\text {th }}$ term [1].

\section{Conclusions}

In this paper, the combined form of the El-Zaki transform and variation iteration method (VITM) was applied to solve different non-linear partial differential equations such as the two-dimensional coupled Burger's equation and the sine-Gordon equation. The system of Burger's equation may be considered as a normal model of sedimentation and evolution of scaled volumes concentrations of [62] two kinds of particles in fluid suspensions and colloids under the effect of gravity, while the sine-Gordon application appears in differential geometry. It is noted that the obtained results by using (VITM) are agreed with the exact solution with small error as shown in Tables 1, 2, 3 and 4. While these result are better than the result obtained by LDM, EHPM, While Table 5 show the proposed method give better result from iteration one but MDM, HPM, give the same result in iteration (4), the MHPIM gave the same result iteration (3). The proposed method shows its capability for solving NPDEs efficiency.

\section{References}

[1] Brezis H. and Browder F.; "Partial differential equations in the $20^{\text {th }}$ century", Advances in mathematics, 35, 76-144, Apr 15, 1998.

[2] Smith G. D.; "Numerical Solution of partial differential equations: finite difference methods", Oxford: Oxford University Press, 1978.

[3] Strang G. and Fix G.; "An analysis of the finite element method", Englewood Cliffs, Prentice, Hall, 188-189, 1973.

[4] LeVeque R.; "Finite volume methods for hyperbolic problems", Cambridge: Cambridge University Press, 2002.

[5] Banerjee P. K.; "Boundary element methods in engineering", London: McGraw-Hill, 1994.

[6] Liu G. R.; "Mesh free methods", Singapore: CRC Press, 2002.

[7] Gottlieb D. and Orzag S.; "Numerical analysis of spectral methods", Theory and Applications. Philadelphia: SIAM, 1977.

[8] Wadati M.; Sanuki H. and Konno K.; "Relationships among Inverse Method, Bäcklund. Transformation and 


\section{Al-Nahrain Journal of Science}

ANJS, Vol.24 (2), June, 2021, pp. 41-47

an Infinite Number of Conservation Laws," Prog. Theor. Phys.53- 419, 1975.

[9] Ablowitz M. J., and Clarkson P. A., "Solutions, nonlinear evolution equations and inverse scattering", University Press, Cambridge, 1991.

[10] Coely A., et al. (Eds.); "Backlund and darboux transformations", American Mathematical Society, Providence, RI, 2001.

[11] Gardner C. S.; Green J. M.; Kruskal M. D. and Miura R. M.; "Method for solving the Korteweg-deVries equation", Phys. Rev. Lett. 19, 1967.

[12] Malfeit W.; "Solitary wave solutions of nonlinear wave equations", Amer. J. Phys. 60-650, 1992.

[13] Yan C. T.; "A simple transformation for nonlinear waves", Phys. Lett. A, 224-77, 1996.

[14] Hirota R.; "Exact solution of the Korteweg-deVries equation for multiple collisions of solitons", Phys. Rev. Lett., 27-1192, 1971.

[15] Cole J. D., "On a quasilinear parabolic equation occurring in aerodynamics", Quart. Appl. Math., 225236, 1951

[16] Burger J. M.; "A mathematical model illustrating the theory of turbulence", Advances in Applied Mechanics, Volume I. Academic Press, New York, 171-199, 1948

[17] Hopf E.; "The partial differential equation $u_{t}+u u_{x}=$ $u_{x x}$ ", Communications on Pure and Applied Mathematics; 3: 201-230, 1950.

[18] Esipov S. E.; "Coupled Burger's equations: A model of polydispersive sedimentation", Phys. Rev. E., 52: 3711-3718, 1995

[19] Kaya D.; "An explicit solution of coupled viscous Burgers' equations by the decomposition method", JJMMS 27(11), 675, 2001.

[20] Abdou M. A. and Soliman, A. A.; "Variational iteration method for solving Burger's and coupled Burger's equations", Journal of Computational and Applied Mathematics, 181(2), 245-251, 2005.

[21] Soliman A. A.; "The modified extended tanhfunction method for solving Burgers-type equations", Physica A, 361, 394, 2006.

[22] Reza A. and Borhanifar A.; "Numerical study of the solution of the Burger's and coupled Burger's equations by a differential transformation method", Computers and Mathematics with Applications, 59, 2711-2722, (2010).

[23] Mittal R. C. and Geeta A.; "Numerical solution of the coupled viscous Burgers' equation", Commun. Nonlinear Sci. Numer. Simul., 16, 1304-1313, 2011.

[24] Vineet K. S.; Tamsir M.; Mukesh K. A. and Sarita S.; "One-dimensional coupled Burgers' equation and its numerical solution by an implicit logarithmic finitedifference method", AIP Advances 4, 037119; https://doi.org/10.1063/1.4869637, 2014.

[25] Al-Fayadh A. and Khawwan H. A.; "Variational iteration transform method for solving Burger and coupled Burger's equations", ARPN Journal of Engineering and Applied Sciences, 12(23), Dec. 2017.

[26] Jain P. C. and Holla D. N.; "numerical solutions of coupled Burger's equation", Int., J. Non-Linear Mechanics, 13, 213-222, 1978.

[27] Arminjon P. and Beauchamp C.; "Numerical solution of Burger's equations", International Journal for Numerical Methods in Engineering; 12, 415-428, 1978.

[28] Fletcher C. A.; "Generating exact solutions of the two-dimensional Burger's equations", International Journal for Numerical Methods in Fluids; 3, 213216, 1983.

[29] Bahadir A. R.; "A fully implicit finite-difference scheme for two-dimensional Burger's equations", Applied Mathematics and Computation; 137, 131137, 2003.

[30] Khater A. H.; Temsah R. S. and Hassan M. M.; "A Chebyshev spectral collocation method for solving Burger's-type equations", Journal of Computational and Applied Mathematics, 222, 333-350, 2008.

[31] Wazwaz, A. M.; "Multiple kink solutions for Mcomponent Burgers equations in $(1+1)$-dimensions and (2+1)-dimensions", Applied Mathematics and Computation, 217(7), 3564-3570, 2010.

[32] Biazar J. and Aminikhah H.; "Exact and numerical solutions for non-linear Burger's equation by VIM", Mathematical and Computer Modeling 49, 13941400, 2009.

[33] Wenyuan L.; "A fourth-order finite-difference method for solving the system of two-dimensional Burger's equations", Int. J. Numer. Meth. Fluids; 64, 565-590, 2010.

[34] Zhao G.; X. Yu and Zhang R.; "The new numerical method for solving the system of two-dimensional Burger's equations", Computers and Mathematics with Applications, 62, 3279-3291, 2011.

[35] Liu F. and Shi W.; "Numerical solutions of twodimensional Burger's equations by lattice Boltzmann method", Commun Nonlinear Sci Numer Simulat, 16, 150-157, 2011.

[36] Alev K. and Ahmet Y.; "An efficient numerical method for solving coupled Burger's equation by combining homotopy perturbation and Pade techniques", Numer. Methods Partial Differential Equations, 27, 982-995, 2010.

[37] Aminikhah H.; "A new efficient method for solving two-dimensional Burger's equation", ISRN Computational Mathematics, Volume, Article ID 603280, 8 pages, doi:10.5402/2012/603280, 2012.

[38] Vaghefi M.; Boveiri S. and Fiouz A. R.; "The solution of two dimensional and time dependent Burger equations using finite difference method", Asian Journal of Engineering and Technology (ISSN: 2321-2462), 02(06), December 2014. 


\title{
Al-Nahrain Journal of Science
}

\author{
ANJS, Vol.24 (2), June, 2021, pp. 41-47
}

[39] Khan M.; "A novel solution technique for two dimensional Burger's equation", Alexandria Engineering Journal, 53, 485-490, 2014.

[40] Sirendaoreji S. J.; "A direct method for solving sinhGordon type equation", Phys. Lett. A, 298, 133-139, 2002.

[41] Whitham G. B.; "Linear and nonlinear waves", Wiley-Interscience, New York, 1999.

[42] Bratsos A. G. and Twizell E. H.; "A family of parametric finite-difference methods for the solution of the sine-Gordon equation", Appl. Math. Comput., 93, 117-137, 1998.

[43] Kaya D.; "A numerical solution of the sine-Gordon equation using the modified decomposition method", Appl. Math. Comput., 143, 309-317, 2003.

[44] Wang Q.; "An application of the modified Adomian decomposition method for $(\mathrm{N}+1)$-dimensional sineGordon field", Appl. Math. Comput., 181, 147-152, 2006.

[45] Wazwaz A. M.; "Exact solutions for the generalized sine-Gordon and the generalized sinh-Gordon equations", Chaos Solitons Fractals, 28, 127-135, 2006.

[46] Lu J.; "An analytical approach to the sine-Gordon equation using the modified homotopy perturbation method", Computers and Mathematics with Applications, 58(11-12), 2313-2319. https://doi.org/ 10.1016/j.camwa., 2009.

[47] Chowdhury M. S. H. and Hashim I.; "Application of homotopy-perturbation method to Klein-Gordon and sine-Gordon equations", Chaos, Solitons and Fractals, 39(4), 1928-1935, 2007.

[48] Batiha B.; Noorani M. S. M. and Hashim I.; "Approximate analytical solution of the coupled sineGordon equation using the variational iteration method. Physica Scripta, 76(5), 445-448, 2007.

[49] Al-Fayadh A. and Faraj D. S.; "Combined Laplace transform-variational iteration method for sineGordon equation", Sci. Int. (Lahore), 31(1), 61-64, 2019.

[50] Mohyud-Din S. T.; Noor M. A. and Noor K. I.; "Modified variational iteration method for solving sine-Gordon equations", World Appl. Sci. J., 6: 9991004, 2009.

[51] Kaya D.; "A numerical solution of the sine-Gordon equation using the modified decomposition method", Appl. Math. Comput., 143(2-3): 309-317, 2003.

[52] Yücel U.; "Homotopy analysis method for the sineGordon equation with initial conditions", Appl. Math. Comput., 203(1): 387-395, 2008.

[53] He, J. H.; "A new approach to nonlinear partial differential equations", Communications in Nonlinear Science and Numerical Simulation, 2, 230-235, 1997.

[54] He, J. H.; "A variational iteration approach to nonlinear problems and its applications", Mechanical Application, 20, 30-31, 1998.
[55] He J. H.; "Variational iteration method-a kind of nonlinear analytical technique: some examples", International Journal of Non-Linear Mechanics, 34, 699-708, 1999

[56] He J. H.; "Variational iteration method-some recent results and new interpretations", Journal of Computational and Applied Mathematics, 207, 3-17, 2007.

[57] He J. H.; "Variational iteration method for autonomous ordinary differential systems", Applied Mathematics and Computation, 114, 115-123, 2000.

[58] Marinca V.; "An approximation solution for onedimensional weakly nonlinear oscillations", Int. J. Nonlinear Sci. Numer. Simul., 3, 107-120, 2002.

[59] Elzaki T. M.; "The new integral transform "Elzaki transform", Global Journal of Pure and Applied Mathematics, 7(1), 57-64, 2011.

[60] Elzaki T. M.; "The new integral transform "ELzaki transform", January 2011.

[61] Elzaki T. M.; Elzaki S. M. and Elnour E. A.; "On the new integral transform "ELzaki transform" Fundamental Properties Investigations and Applications", Global Journal of Mathematical Sciences: Theory and Practical, 4(1), 1-13, 2012.

[62] Kaya D.; "A numerical solution of the sine-Gordon equation using the modified decomposition method", Applied Mathematics and Computation, 143(2-3), 309-317, 10 November 2003.

[63] Saeed R. K. and Mustafa A. A.; "Numerical solution of Fisher-KPP equation by using reduced differential transform method" AIP Conference Proceedings, 1888(7), 2017. 